Harnessing the activity of the fungal metalloprotease, Mpr1, to promote crossing of nanocarriers through the blood-brain barrier.

Phylicia A. Aaron and Angie Gelli

Supporting Information

(3 pages and 3 figures) 


\section{Standard Curve}

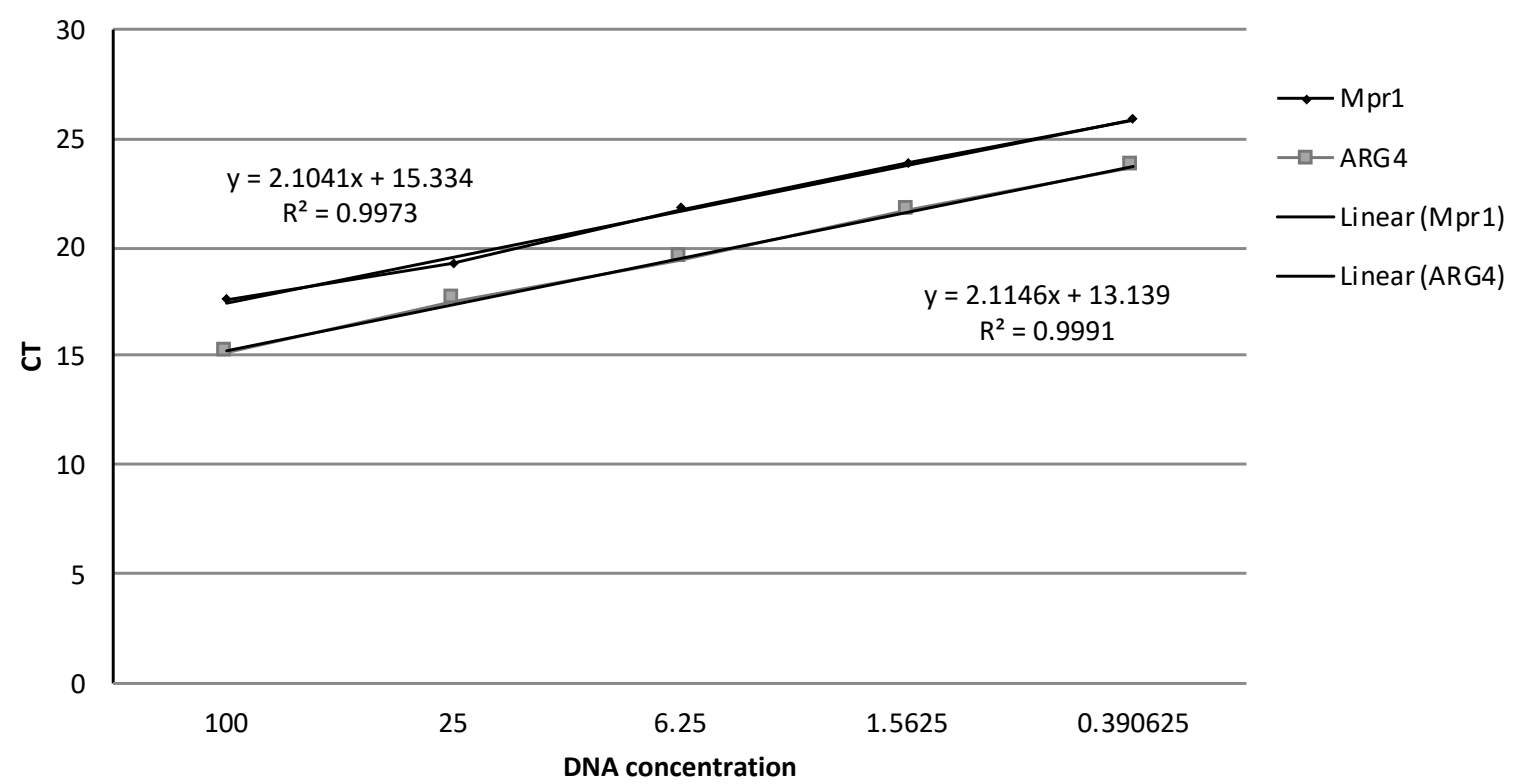

\begin{tabular}{|l|l|}
\hline Strain & Copy Number \\
\hline H99 & 1 \\
\hline P. pastoris & 0 \\
\hline P. pastoris $<$ CnMpr1 ${ }^{\text {HIS6 }}>$ & $2-3$ \\
\hline
\end{tabular}

Figure S1, quantitative PCR 
${ }^{576}$ DGDLEAGIVIHEYSHGLSTR ${ }^{595}$

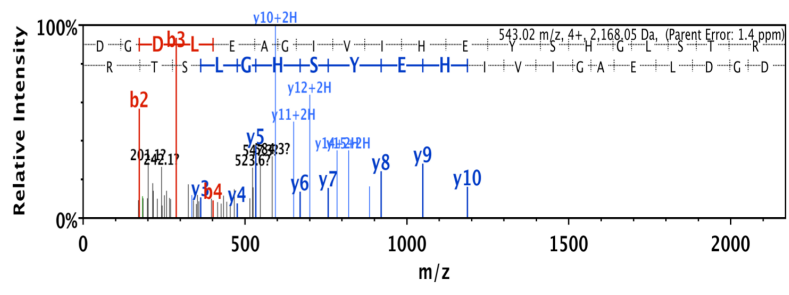

${ }^{596}$ LTGGPANSGCLGYGEAGGMGEGWGDAIATLIR $^{627}$

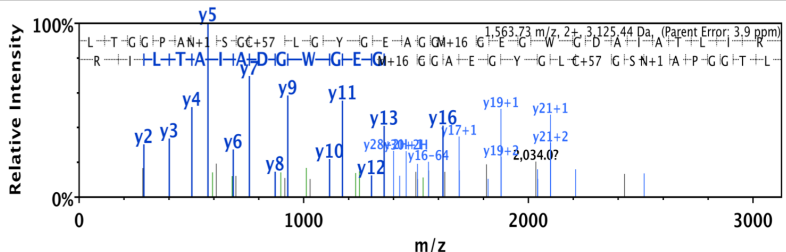

$m / 2$

000

\begin{tabular}{|c|c|c|c|c|c|c|c|c|c|}
\hline & $B+2 F$ & B-NH & & AA & Ylons & $\mathrm{Y}+2 \mathrm{H}$ & $\mathrm{Y}-\mathrm{NH}_{3}$ & & \\
\hline 116.0 & 58.5 & & 98.0 & D & $2,169.1$ & $1,085.0$ & 2.152 .0 & $2,151.0$ & 20 \\
\hline 173.1 & & & 155.0 & G & 2.054 .0 & 1.027 .5 & $2,037.0$ & 2.036 .0 & \\
\hline 288.1 & 144.5 & & 270.1 & D & 1.997 .0 & 999.0 & $1,980.0$ & $1,979.0$ & \\
\hline 401.2 & 201.1 & & 383.2 & L & $1,882.0$ & 941.5 & $1,865.0$ & $1,864.0$ & \\
\hline 530.2 & 265.6 & & 512.2 & E & $1,768.9$ & 885.0 & 1.751 .9 & $1,750.9$ & 6 \\
\hline 601.2 & 301.1 & & 583.2 & A & $1,639.9$ & 820.4 & $1,622.8$ & $1,621.8$ & 5 \\
\hline 658.3 & 329.6 & & 640.3 & G & $1,568.8$ & 784.9 & 1.551 .8 & 1.550 .8 & 4 \\
\hline 771.4 & 386. & & 753.3 & 1 & 1.511 .8 & 756.4 & 1.494 .8 & 1.493 .8 & 3 \\
\hline 870.4 & 435 & & 852.4 & v & 1.398 .7 & 699.9 & $1,381.7$ & 1.380 .7 & 12 \\
\hline & & & & & & & & & \\
\hline & & & $1,102.6$ & H & $1,186.6$ & 593.8 & $1,169.5$ & $1,168.5$ & \\
\hline & & & & & .5 & 525.3 & $1,032.5$ & $1,031.5$ & \\
\hline 1.412. & 706 & & 1.39 & & 920 & 460.7 & & 902.4 & \\
\hline & 75 & & & & 757 & 379 & 740.4 & 4 & \\
\hline .63 & 818 & & 1,618 & H & 67 & 335.7 & 653.3 & 652.4 & \\
\hline .69 & 84 & & 1.675 & & 53 & 26 & $\overline{51}$ & 515.3 & \\
\hline 1,80 & 90 & & & L & & & & 3 & \\
\hline & & & & & & & 2 & .2 & \\
\hline & 998 & & & & & & 59.1 & 258.2 & \\
\hline & & & .151 .0 & & 17 & 88.1 & 158. & & \\
\hline
\end{tabular}

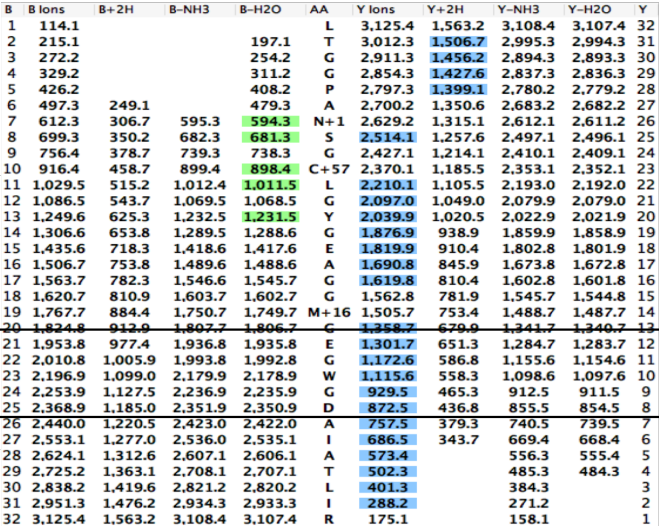

Figure $\mathrm{S} 2, \mathrm{~ms} / \mathrm{ms}$ spectral analysis 


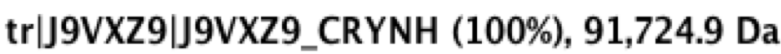

Extracellular elastinolytic metalloproteinase OS=Cryptococcus neoformans var. grubii serotype A (strain H99 / ATCC 208821 / CBS 1051 57 exclusive unique peptides, 105 exclusive unique spectra, 1792 total spectra, $370 / 831$ amino acids (45\% coverage)

M R S A L I A L L $L D D A V H V F E P$

R T G V T H I F A R

I HSS S S G E T E

$S$ L P L G E V H H

L P R L S P I D D L

V S D V S S_A_R L M Y

A S D F D I D E L R

L S V V T E P W D T

VYAHE NWEGR

Y T A N M Y H D L L

N F L T P P D G Q A

A N S G C L G Y G E

S N R G I R N Y K Y

V E K Y G F G P T L

Q L I V D A M K L Q

D A A V V G T P W
$P F L A T L T A A R$ $R G L \mid D E P$ | D V $Q L L N G L E V S D$ K V C T T L H Q H L E I K E T H K S M R E D I S P F D L T S T Q V S E G A R L D K I E M M K G G K VAS P L GWH S F N N F L L N Y R P T Y R L G F D E L S G PR M R M Y I W D T A G G M G E G W G D $S$ T N F T I N P S T F P P T D T S K H N PCR P S F F D A R G G G V R S D G F K
P H H R D K H S A $K R \vee A Q T F L G S$ $G D|N L N| D R D$ D E H K A H L A L H I E N H L R A M C T P H H T L K P K P VWKYE V E M K D Q K P L P I P P K K P T S A N P W D V T N D S H I F V Y E Y N F Q A Y N F R L G A T P Y R D G D L E A I A T L I R Q V E Y K T L D K P G Y W D Y Y T R T S E E S N A I I Q A D Q I L V PKK V C E S K K
SR TRK S L SF G $Q$ L G A E G E F $G R$ I $M$ S W N S F K G E T G I W G L V D O P A V S T O S M A F A E P P T E V I $S W Y E A Y V D V L$ I Q P Y S Y Q V F P I $P$ GE T T N H N Y G E P E G L A P K E G K G G D P V V C N A G I V I H E Y S H E H K N F E N G T D G V H A I G E VW A V D A A G R P L P L T G G E N A C L I W A
P A H S H A S F V $Y$ I R E D S Y T D A H P G S V P S L S D K S A A Q V V L G S L S P V E A L V S L $S$ G A A L S K A G V $S G E L I R V V D W$ W G I N D P V S G N $T V F N T T A G N N$ Y V D M V V T Q L F A Q D G S G Y N N A G L S T R L T G G P V F S M G A W A A N E F L F V L S Q R L V P K Y G N A L A I Q A F A E R G L E

= predicted cleavage site for the prodomain

Figure S3, amino acid sequence of Mpr1 protein from Cryptococcus neoformans var. grubii (H99) 